\title{
REVIEW
}

\section{Targeting the mucosa: genetically engineered vaccines and mucosal immune responses}

\author{
L Stevceva $^{1}$, AG Abimiku ${ }^{2}$ and G Franchini ${ }^{1}$ \\ ${ }^{1}$ National Cancer Institute, Basic Research Laboratory, Bethesda, MD 20892, USA; ${ }^{2}$ Institute of Human Virology, University of \\ Maryland, Baltimore, MD 21201, USA
}

\begin{abstract}
The discovery that inoculation of DNA leads to strong and long lasting immune responses generated enthusiasm to assess the efficacy of various genetically engineered vaccines against mucosally acquired infections. Various techniques have been used to generate the most suitable DNA vaccines, ranging from immunization with naked DNA to utilizing genetically engineered recombinant viruses and bacteria to deliver the DNA. Different DNA vaccine modalities and mucosal immune responses to them have been discussed. It has been shown that even though intramuscular and intradermal immunization with these vaccines generates strong systemic responses, mucosal responses are not induced. It has been proposed that the site of immunization determines mucosal immune responses and that primed lymphocytes preferentially accumulate at sites where they have been induced thus generating the strongest cellular and antibody responses at the site of vaccination. The impact of the site of induction on mucosal immune responses to vaccines is discussed. It is possible to enhance desired vaccine effects in the mucosa and to modify the undesirable side effects. Cytokines such as IL-2, IL-12, IL-15 and IL-18 have been used to enhance CTL activity while IL-5, IL-6 and the chemokine MIP-1 $\alpha$ have shown the capacity to increase IgA responses to vaccines. Genes and Immunity (2000) 1 , 308-315.
\end{abstract}

Keywords: vaccine; naked DNA; mucosa; CTL; IgA

\section{The mucosal immune network: importance of the site of antigen presentation}

Next to the skin, human mucosa is the largest organ of the human body that is continuously exposed to outside antigens, microorganisms and viruses. It represents an area of $400 \mathrm{~m}^{2}$ that is well equipped to deal with daily insults by infectious agents and allergens. Therefore, its importance in the immune defense system of the body is immense. Mucosa is often the first line of defense against infection and only when the mucosal barrier breaks down infection may occur. Consequently, in the generation of suitable vaccines against pathogens whose main entry route is the mucosal layer, it is very important to assess mucosal immune responses to immunization.

The mucosal defense system is very complex. Immunoglobulin $\mathrm{A}$ and $\mathrm{M}(\operatorname{Ig} \mathrm{A}, \operatorname{Ig} \mathrm{M})$ are believed to be major players and their role has been elucidated in various systems. It is known that IgA on mucosal surfaces prevents mucosal attachment of bacteria and viruses thus limiting their entry. In addition, both IgA and IgM are important in the so-called mechanism of 'immune exclusion' where antigens are captured during their intraepithelial transport, neutralized and excreted thereby preventing

Correspondence: Liljana Stevceva, Division of Basic Sciences, National Cancer Institute, Building 41, Bethesda, Maryland, 20892, USA.

E-mail: stevcevl@mail.nih.gov

Received and accepted 1 February 2000 mucosal damage. ${ }^{1}$ The role of IgG antibodies is less defined and it is thought that they may have a role in protecting the female genital tract and the respiratory tract where they can be excreted by passive paracellular transport to neutralize antigens in the lumen. ${ }^{2,3}$ Novel findings, however, that passive transfer of human antibodies against HIV-1 of IgG1 subtype was able to protect macaques against intravenous and intravaginal challenge with SHIV indicate that IgG antibodies following passive tranfer can neutralize the virus ${ }^{4,5}$ and blunt viral infection. Furthermore, administration of a cocktail of IgG1 antibodies against human HIV-1 to pregnant macaques prevented neonatal transmission and protected the mothers from intravenous infection. ${ }^{6}$ Caution however has been advised in interpreting the results since it is not clear if the same effect can be achieved by using more virulent viral strains or whether the virus was only suppressed. ${ }^{7}$

It has been proposed that the site of induction determines mucosal immune responses and that primed lymphocytes preferentially accumulate at sites where they have been generated. ${ }^{8}$ Published data have shown that it is possible to induce strong humoral and cellular immune responses by injecting naked DNA intramuscularly, subcutaneously or by a novel jet delivery. However, even though naked DNA vaccines utilizing these routes of immunization generates systemic immune responses sufficient to confer protection, mucosal immunity is not induced. In fact, intramuscular immunization of BALB/c and C57Bl6 mice with naked plasmid DNA (pRc/Yhsp60) encoding Yersinia enterocolitica 60-kDa heat 
shock protein (Y- HSP60) conferred protection against dissemination of this infection in the spleen but not at the site of mucosal entry. ${ }^{9}$

In regards to $B$ cells, the proposed model, based on numerous studies looking into B-cell migration patterns and adhesion molecule expression suggests that primed lymphoid cells from nasal-associated lymphoid tissue (NALT), from Eustachian tube-associated lymphoid tissue (TALT) and bronchus-associated tissue (BALT) preferentially home into nasal and salivary glands, pharyngeal and middle ear mucosa, bronchial and mammary glands, but can also home into urogenital tract. For example, intranasal immunization with a DNA vaccine constructed with the CMV promoter conjugated to env gp160 and rev genes in liposomes induced high levels of mucosal IgA antibodies in feces and vaginal fluids that were able to neutralize HIV-1. ${ }^{10}$

Primed B cells from Payer's patches (gut-associated lymphoid tissue, GALT) preferentially home into small intestinal mucosa but can also home into the urogenital tract. This was demonstrated in a study where oral immunization with a rotavirus VP6 DNA vaccine encapsulated in polylactide-coglycolide (PLG) microparticles was able to elicit both rotavirus-specific serum antibodies and intestinal immunoglobulin A (IgA). After challenge at 12 weeks postimmunization with homologous rotavirus, fecal rotavirus antigen was significantly reduced compared with controls. Earlier and higher fecal rotavirus-specific IgA responses were noted during the peak period of viral shedding, suggesting that protection was due to specific mucosal immune response. ${ }^{11}$

$\mathrm{B}$ cells induced in appendix and colonic-rectal solitary follicles (also part of GALT) preferentially home into large intestinal mucosa and the urogenital tract. ${ }^{12-14}$ What that means in terms of mucosal vaccines would be that, intrarectal immunization, for example, would induce high levels of secretory $\operatorname{IgA}$ in large intestinal mucosa, some in the small intestine and urogenital tract and none in the mucosa of the airways and mammary glands.

It would appear that the same concept applies to B cells originating from vaginal mucosa. Intravaginal immunization with a DNA plasmid encoding the HIV-1 envelope (pcMN160) induced production of Ag-specific intravaginal immunoglobulins that were able to neutralize HIV-1 in vivo. ${ }^{15}$ In another study, immunization was induced by using gene gun technology via vaginal mucosa, Payers patch or abdominal skin. The results showed that even though immunization via either route induced vaginal antibodies, vaginal antibody titers of $\operatorname{IgA}$ and $\operatorname{IgG}$ were only consistently sustained if elicited via vaginal route. ${ }^{16}$

Even though the same general rule appear to apply to $\mathrm{T}$ cells as well, the situation is much more complex. While activated $\mathrm{T}$ cells re-enter lymphoid tissues and preferentially accumulate in the lymphoid tissue where the initial activation took place, memory $\mathrm{T}$ cells migrate continuously and randomly, similar to naïve $\mathrm{T}$ cells. ${ }^{17,18}$ The implication in terms of vaccine development would be that, in the initial phase of an immune response, once primed, Ag-specific T cells would randomly enter and leave various lymphoid compartments but would be preferentially retained in the lymph node where the antigen was presented at first. ${ }^{19}$

\section{Inducing mucosal immunity by various DNA vaccine modalities}

The discovery that inoculation of naked DNA leads to long-lasting and strong immune responses had generated enthusiasm to assess this vaccine modalities in mice as well as in higher mammalian species (Table 1).

\section{Immunization with naked DNA}

DNA can be introduced to the body by a simple intramuscular or intradermal injection or by propelling DNAcoated particles in various tissues, ${ }^{20}$ most commonly the skin. Professional antigen presenting cells (APCs) are essential for priming immune responses, either through direct transfection of APC, ${ }^{21}$ cross-priming ${ }^{22}$ or both. The DNA is transcribed and the RNA translated into the host and the degradation of the protein in the proteosome generates peptides that are presented in the context of MHC Class I molecules. Even though the inoculation leads to transfection of a limited number of cells, it still initiates powerful responses. Intradermal and intramuscular gene vaccination of mice induces antigen-specific Th1 cells that secrete high levels of IFN $\gamma^{23,24}$ and stimulates production of antibody of the IgG2a isotype ${ }^{24,25}$ while immunization with soluble protein antigens induces predominantly IgG1 and IgE antibodies and Th2 cells that secrete IL-4 and IL-5. It has also been shown that DNA vaccination appears to be highly effective in inducing $\mathrm{MHC}$ Class I-restricted, $\mathrm{CD}^{+}$cytotoxic $\mathrm{T}$ lymphocyte (CTL) responses in mice as well as in nonhuman primates. CTL responses induced by DNA vaccination can last for more than 1 year. ${ }^{26}$ The effect of DNA vaccination is dominant and it has been shown that preimmunization with DNA prevents subsequent induction of Th2 responses and induction of antigen specific IgE when a protein in alum is given. ${ }^{23}$ While intradermal and intramuscular injection of naked DNA induces predominantly Th1 response, gene immunization of mice with bolistic delivery into the skin with DNA-coated gold microspheres induces a Th2 response. ${ }^{27}$ It is believed that in this case the small amount of DNA transfected is insufficient to provide the adjuvant effect necessary for induction of Th1 response. Naked DNA is increasingly tested as a vaccine against infectious diseases but it was also used in immunizing against cancer and inducing tolerance to allergens. ${ }^{28,29}$

ACA mice immunized with plasmid DNA expressing the Epstein-Barr virus (EBV) nuclear Ag-4 (EBNA-4) were partially protected against syngeneic mammary carcinoma line (S6C) expressing EBNA-4 (S6C-E4). This protection was enhanced by co-immunizing mice with EBNA-4 and GM-CSF-expressing plasmids and a full protection was achieved by co-immunizing mice with EBNA-4 and IFNg-expressing plasmids. Mice that have rejected the EBNA-4-positive tumor were also resistant against a subsequent challenge with the original nontransfected tumor line. This response was long-lived as it was found that even 3 months after the last immunization, full protection was achieved. ${ }^{28}$

A very interesting study used nanoparticles containing DNA coding for a major peanut allergen (Arah2) complexed with chitosan, a natural polysaccharide derived from crustacean shells to orally feed mice rendered allergic to peanut. Chitosan served as delivery vehicle promoting adhesion to the mucosa and transport through 
Table 1 Immune responses to genetically engineered vaccines

\begin{tabular}{|c|c|c|c|}
\hline Antigen & Vector & Immunization route & Effects \\
\hline $\begin{array}{l}\text { Glutathione S-transferase of } \\
\text { Schistosoma haematobium }\end{array}$ & $\mathrm{rBCG}$ & i.n.; i.p. & $\begin{array}{l}\text { Neutralizing antibodies of IgG1, IgG2a, } \\
\operatorname{IgG} 2 \mathrm{~b} \text { and } \operatorname{IgA} \text { in serum and IgA in } \\
\text { bronchoalveolar fluids }\end{array}$ \\
\hline SIVmac251env, gag, nef & $\mathrm{rBCG}$ & oral & $\begin{array}{l}\text { CTL responses in Payer's patches, } \\
\text { intestinal mucosa }\end{array}$ \\
\hline Escherichia Coli $\beta$ galactosidase & Shigella Flexneri & & Strong cellular and humoral response \\
\hline $\begin{array}{l}\text { Escherichia Coli } \beta \text { galactosidase and } \\
\text { Lysteria } \\
\text { Monocytogenes actA and hly genes }\end{array}$ & $\begin{array}{l}\text { Salmonella } \\
\text { Typhimurium }\end{array}$ & oral & $\begin{array}{l}\text { Strong CTL and proliferative } \\
\text { responses in spleen and mesenteric } \\
\text { lymph nodes and specific antibodies in } \\
\text { sera }\end{array}$ \\
\hline
\end{tabular}

Fragment $\mathrm{C}$ of tetanus toxin

Onchocerca Volvulus glutathione S transferase

Rotavirus VP6 DNA encapsulated in polylactide-coglycolide microparticles

Plasmid DNA encoding Yersinia Enterocolitica heat shock protein (YHSP60)

Plasmid DNA encoding env GP160 and rev of HIV-1 virus in liposomes

Plasmid DNA encoding human growth hormone under the control of cytomegalovirus promoter

Env and gag genes of HIV-1

SIV gag-derived multicytotoxic Tlymphocyte epitope

SIV protein, p17 (gag) and gp41 (env)

DNA encoding for peanut allergen (Arah2) complexed with chitosan

Melanocyte differentiation antigens: GP100, MART-1, tyrosinase and tyrosinase-related proteins (TRP-1 and TRP-2)

Human myelin basic protein (hMBP) or hMBP as a fusion protein with $\beta$-glucuronidase

EBNA-4 gene of Epstein-Barr virus

Melan-A (membrane protein expressed in melanosomes and endoplasmatic reticulum)

SIV gag, pol, env genes

HIV-1 gag, pol, env genes

Rabies virus glycoprotein of the ERA strain

Plasmid DNA encoding HIV-1

envelope (pcMN160)
Typhimurium

Salmonella

Typhimurium

oral

oral

i.m.

i.n.

Intravaginal, intradermal

(abdominal skin),

Payer's patches

Naked DNA priming, boosting with rFowl poxvirus

Priming with DNA,

boosting with rMVA

Recombinant

poliovirus

i.m.; gene gun into

abdominal epidermis

i.n.; i.v.

oral

Recombinant vaccinia i.v virus

Lactobacillus casei with parenteral surface-anchored tetanus toxin

fragment C

i.m.; gene gun

Recombinant vaccinia virus

intraperitoneal

Recombinant poxvirus (NYVAC)

Recombinant

canarypox

Replication-defective

adenoviral

recombinant
Strong proliferative responses in PBMCs and spleen; specific antibodies in sera

Rotavirus specific serum antibodies and intestigal $\operatorname{IgA}$, reduction of fecal rotavirus antigen

Protection against disseminated infection in spleen but not at mucosa sites

High levels of mucosal IgA antibodies in feces and vaginal fluids able to neutralise HIV

Ag-specific $\operatorname{IgA}$ and $\operatorname{IgG}$ antibody titers consistently sustained only via vaginal route

Protection from i.v. challenge with rFPV expressing HIV-1 antigen and i.v. challenge with HIV-1

Induction of CTL's, no protection after intrarectal challenge

Strong IgA antibodies in rectal and vaginal secretions, IgG antibodies in sera, anti-SIV $\mathrm{T}$ cell proliferative responses

Allergic reaction to peanut Arah2 protein significantly diminished, increased IgA, IgG2a-Th1 type response

Full protection from s.c. challenge with B16 tumor cells, partial protection if injected i.v, vitiligo in mice immunized with rVV encoding in mTRP-1

Secretion of human myelin basic protein (can be used to induce peripheral $\mathrm{T}$ cell tolerance)

Partial or full protection against the outgrow of S6C-E4, a nonimmunogenic tumor transfected with EBNA-4

Melan-A-specific tumor-reactive CTL's efficiently induced upon stimulation of PBMC from melanoma patients. High levels of peptide-specific lysis for splenic $\mathrm{T}$ cells

Protection against i.r. challenge. Increased survival following i.v. challenge

Cross-protection against i.v. challenge with HIV-2

s.c. and i.n. induced serum and mucosal antibodies and i.n. immunization induced higher titres of vaginal and intestinal antibodies of IgA type

intravaginal
Ag-specific intravaginal antibodies able to neutralise HIV in vivo 
the epithelium. In control animals, challenge with Arah2 caused severe anaphylactic reaction with increased plasma histamine concentration, increased vascular permeability and raised peanut-specific serum IgE. This reaction was substantially diminished in animals pretreated with chitosan-DNA particles. It was shown that the immunization may be improving local defense mechanisms by increasing production of fecal IgA and serum IgG2a, decreasing IgE and directing the response to Th1. This study describes a potentially new way to use DNA immunization in inducing tolerance to antigens and may potentially be very useful in oral tolerance to respiratory allergens. The use of chitosan enables oral feeding of the DNA and efficient DNA transfection of epithelial cells. ${ }^{29}$

While the application of DNA vaccines by intramuscular or intradermal inoculation targets a relatively limited amount of antigen presenting cells, delivery of DNA vaccines by carriers could increase the number of transfected APCs in immunologically relevant organs such as the gut and the gut-associated lymphoid tissue increasing the immune response. These strategies may help the development of vaccines that could be administered orally. Both bacteria and viruses have been used as vector carriers to deliver DNA.

\section{Viruses as delivery vectors}

Viruses adhere and penetrate the cell membrane through viral receptors. Recombinant viruses in which foreign DNA is inserted function as vehicle to transport the foreign DNA into the cell. The foreign DNA is expressed by using the cellular machinery and the protein transported through the endogenous pathway of the APCs where it combines with the MHC Class I molecules and is presented to $\mathrm{CD}^{+} \mathrm{T}$ cells. ${ }^{30}$ Various viral carriers have been successfully used and have shown capacity to elicit strong immune responses. Members of the poxvirus family such as attenuated vaccinia derivative and avipox viruses are the most widely used. Vaccinia recombinant and its derivative have a broad host range and have been used for the development of human and animal vaccines. Recombinant vaccinia virus is used as an effective oral wildlife rabies vaccine ${ }^{31}$ and is currently investigated as an HIV vaccine.

Consecutive immunization involving priming with DNA and boosting with recombinant Fowl pox virus (rFPV) vaccines encoding common HIV-1 antigens induced greater HIV-1-specific immunity in mice than both vectors alone and protected them from challenge with a recombinant vaccinia virus expressing HIV-1 antigens. This vaccine regimen also protected macaques from an intravenous HIV-1 challenge. ${ }^{32}$ High levels of CTLs specific for a single simian immunodeficiency virus (SIV) gag-derived epitope were elicited in rhesus macaques by using a multicytotoxic T- lymphocyte (CTL) epitope gene and vaccination regimen using priming with DNA and boosting with recombinant modified virus Ankara (MVA; an attenuated vaccinia virus). These vaccineinduced CTLs were capable of killing SIV-infected cells in vitro. As shown by FACS analysis of tetrameric major histocompatibility complex-peptide complexes, the vaccinated animals had 1 to $5 \%$ circulating CD ${ }^{+}$lymphocytes specific for the vaccine epitope, frequencies comparable to those in SIV-infected monkeys. However, upon intrarectal challenge with pathogenic SIVmac251, no evidence for protection was observed in at least two of the three vaccinated animals. ${ }^{33}$ A different study described enhanced responses with an optimized vaccine regimen that primes with DNA-Env, boosts with recombinant vaccinia virus-Env, and re-boosts with purified Env proteins. ${ }^{34}$

Recombinant vaccinia virus is the most widely used vector in design of antitumor vaccines. Immunization against self antigens such as melanocyte differentiation antigens encoded in rVV was shown to induce full protection in mice challenged with tumor cells. ${ }^{35}$ Unfortunately, this vaccine also induced vitiligo-like symptoms. Recombinant vaccinia virus (rvv-GM-CSF) expressing the granulocyte macrophage stimulating factor (GM-CSF) as tumor vaccine was evaluated in the murine B16-F10 melanoma model. The vaccine was prepared by infection of irradiated tumor cells with rvv-GM-CSF. Immunization of C57BL/6 mice with this construct inhibited tumor development, lung metastasis and prolonged survival. The effect was tumor-cell specific. In another study, strong tumor-specific CTL responses were elicited by using recombinant vaccinia encoding amino acid sequences corresponding to natural CTL-defined peptides from tumor antigens such as melan-A peptide analogue. The use of mutated minigene construct lead to improved antigen recognition and enhanced immunogenicity. ${ }^{36}$

The avipoxvirus has limited host range and its replication is abortive in mammalian cells. This property has been exploited to generate a safe live attenuated vaccine for humans. ${ }^{37}$ The most widely tested to date has been canarypox virus-based vaccine that was able to crossprotect not only against HIV-1 but also against HIV-2 viruses in macaque models and is currently undergoing phase 2 trials. $^{38-41}$

Adenoviruses that replicate in the gut have great potential in eliciting mucosal immune responses and were proven very effective and safe as vectors for viral vaccines. The antigenic diversity (over 45 serotypes described) allows the exploitation of antigenically distinct vectors for multiple sequential booster immunizations. Recombinants using adenovirus as vector have been described and used for a variety of animal viruses including hepatitis B, hepatitis C, vesicular stomatitis, herpes simplex, rabies, parainfluenza and HIV. ${ }^{42-44}$ Adenoviral vectors have also been used to induce antitumor immunity. High efficiency transfection of IL-2 gene into glioblastoma cell lines using adenoviral vectors induced production of high levels of IL-2 and activation of CD8 ${ }^{+} \mathrm{T}$ cells suggesting generation of antitumor response. ${ }^{45}$ Recently published study where replication-defective adenoviral recombinant was used to induce immune responses against rabies, has shown that it is possible to induce good mucosal immune responses using nonreplicative adenoviruses by immunizing intranasally. ${ }^{46}$

Since most HIV-1 infection occurs mucosally, it may be necessary to develop immunization strategies in order to elicit strong mucosal as well as systemic responses and viral recombinants may be a good tool in this endevour. ${ }^{47}$

This issue was addressed in studies that utilise polioviruses as carriers to immunize against SIV (a virus that induces AIDS-like disease in monkeys ${ }^{48}$ ) by mucosal route. Intranasal immunization with this construct elicited strong IgA responses in rectal and vaginal secretions and IgG antibodies in sera. Anti-SIV T cell proliferative 
responses in PBMCs were also induced. ${ }^{49}$ However, efficacy studies for this vaccine are not yet available.

Herpes simplex virus (HSV) that is capable of a single round of infection (DISC-HSV) was used as a vector to elicit antitumor effect in colorectal cancer. Clear antitumor effects in tumor prevention and tumor therapy were shown with DISC-HSV/GM-CSF. ${ }^{50}$

Plasmid DNA vectors that contained replicons derived from prototype alphavirus, Sindbis and Semliki Forest virus were used to enhance immunogenicity of nucleic acid vaccines. These plasmids, when transfected into cells launch a self-replicating RNA vector (replicon) that, in turn, directs the expression of a model tumor antigen. This strategy elicited immune responses at doses 100 to 1000 fold lower than conventional DNA plasmids. ${ }^{51}$

\section{Bacteria as vectors}

Mechanisms by which bacteria deliver DNA to host cells is largely dependent on the property of the bacterium itself. Various bacteria have been recently successfully used to deliver DNA. So far, Mycobacterium Bovis Bacillus Calmette-Guerin, Listeria Monocytogenes, Salmonella Typhimurium and Shigella Flexneri are the ones in which most of the studies have been done.

Most of the bacteria used as vaccine vectors have been engineered to express the desired protein by using prokaryotic promoters. However, other studies have shown that immune responses can also be elicited by inserting plasmid DNA with an eukaryotic promoter so that the bacterium is only a vehicle for the DNA.

rBCG has been successfully used to induce immune responses to glutathione S-transferase from Shistosoma haematobium (Sh28GST), the ethiologic agent of human urinary schistosomiasis ${ }^{52}$ and to SIVmac251env, gag and nef proteins. ${ }^{53}$ After oral ingestion of rBCG SIV nef strain, rBCG entered oropharyngeal and intestinal mucosa, was disseminated to Payer's patches and systemic organs where it multiplied and persisted for a long time inducing CTL responses. Also, large numbers of $\mathrm{rBCG}$ remained in the intestinal lumen inducing Ag-specific proliferation of intra-epithelial lymphocytes. ${ }^{54}$ Epitops from the HIV virus, such as the nef protein $^{55}$ and the Env protein V3 region ${ }^{56}$ have also been used to successfully engineer vaccines using rBCG as a vector.

Attenuated strains of gram-negative bacteria, such as Salmonella Typhimurium and Shigella Flexneri have also been used to deliver DNA. S. Flexneri enters the cell cytosol after phagocytosis and delivers plasmids directly to the intracellular compartment. An invasive phenotype of bacteria is necessary for successful plasmid delivery. Intranasal immunization with Shigella induced strong cell and humoral responses against Escherichia Coli $\beta$-galactosidase. $^{57}$ Similarly, Shigella Flexneri attenuated by deletional mutation of asd gene harboring a DNA measles virus vaccine plasmid can induce strong virus-specific $\mathrm{CD}^{+} \mathrm{CTL}$ responses and IFN-gamma secretion in splenocytes. ${ }^{58}$

S. Typhimurium, on the other hand, remains in the phagosomal compartment of the host cell. ${ }^{59}$ Nonetheless, this bacterium still has the ability to deliver eukaryotic expression vectors not only to macrophages but also to dendritic cells. ${ }^{60,61} \mathrm{~S}$. Typhimurium has been successfully used as a vector to deliver several foreign antigens and to elicit immune responses.

Live Salmonella Typhimurium, attenuated by deletional mutation of the aro $A$ gene causing requirement for aromatic metabolits (SL7207) has been used as carrier for the pCMVbeta vector that contains the $\beta$-galactosidase (betagal) gene, ${ }^{60}$ attenuated Salmonella Typhimurium strain CVD 915 was used to deliver plasmids pTETnir15 and pcDNA3tetC encoding fragment C (Frag C) of tetanus toxin under the control of prokaryotic or eukaryotic promoters ${ }^{62}$ and attenuated Salmonella typhimurium expressing OvGST was also able to induce cellular and humoral responses in mice to Onchocerca volvulus glutathione Stransferase (OvGST). ${ }^{63}$

Attenuated intracellular bacteria can accomplish delivery of DNA to APCs. Dietrich et al ${ }^{64}$ engineered the intracellular pathogen Listeria Monocytogenes to deliver plasmid DNA into the cytosol of macrophages. After inoculation, these bacteria lyse the vacuole of the macrophage and enter the cytosol where they grow. Listeria also has the ability to spread from cell to cell without contacting the extracellular milieu. In this model, immunity is mediated by $\mathrm{CD}^{+} \mathrm{T}$ cells and antibodies do not play a significant role.

In addition, recombinant Lactobacillus casei which expresses surface-anchored tetanus toxin fragment $C$ and secretes human myelin basic protein was generated. It is proposed that such a construct may be useful in inducing periferal $\mathrm{T}$ cell tolerance and protection against autoimmune diseases as well as in oral vaccination against tetanus. ${ }^{65}$

\section{Enhancing and modifying mucosal immune responses}

Several approaches have been used to enhance and/or modify mucosal immune responses.

The potency of Th1 vs Th2 cytokines to enhance immune responses to DNA vaccines was examined in a study where DNA expression construct encoding the HSV-2 gD protein (a glycoptrotein involved in HSV binding and entry into the host cell) was codelivered with the gene plasmids encoding the Th1-type (IL-2, IL-12, IL-15 and IL-18) and Th2-type (IL-4 and IL-10) cytokines by intramuscular injection. Th1 cytokine gene coadministration not only enhanced the survival rate but also reduced the frequency and severity of herpetic lesions following intravaginal HSV challenge. On the other hand, co-injection with Th2 cytokine genes increased the rate of mortality and morbidity of the challenged mice. IL-12 was a particularly potent adjuvant for the gD DNA vaccination. ${ }^{66}$ In a follow-up study, co-injection with IL-12 cDNA and gD DNA vaccine inhibited both systemic gD-specific $\mathrm{Ab}$ and local $\mathrm{Ab}$ levels compared with gD plasmid vaccination alone. In contrast, Th1 cell proliferative responses and secretion of cytokines (IL-2 and IFN $\gamma$ ) and chemokines (RANTES and MIP-1 $\alpha$ ) were significantly increased by IL-12 coinjection. IL-12 coinjection with a gD DNA vaccine showed significantly better protection from lethal HSV-2 challenge compared with gD DNA vaccination alone in both inbred and outbred mice. This enhanced protection appears to be mediated by CD4+ T cells, as determined by in vivo $\mathrm{CD}^{+}$ T cell deletion. ${ }^{67}$

In a study in the macaque model, the gag, pol, and env genes of SIV $(\mathrm{K} 6 \mathrm{~W})$ were expressed in the NYVAC vector, a genetically engineered derivative of the vaccinia virus Copenhagen strain that displays a highly attenuated 
phenotype in humans. ${ }^{68}$ In addition, the human IL-12 p35 and p40 subunits as well as the IL-2 gene, were expressed in separate NYVAC vectors and inoculated intramuscularly, in conjunction with or separate from the NYVACSIV vaccine. The overall cytotoxic T-lymphocyte (CTL) response was greater, at the expense of proliferative and humoral responses, in animals immunized with NYVACSIV and NYVAC-IL-12 than in animals immunized with the NYVAC-SIV vaccine alone. Significantly, five of the 11 vaccinees exposed mucosally to SIV(mac251) showed a transient peak of viremia 1 week after viral challenge and subsequently appeared to clear viral infection. In contrast, all 12 animals inoculated intravenously became infected, but 5 to 6 months after viral challenge, four animals were able to control viral expression and appeared to progress to disease more slowly than control animals. ${ }^{69}$ Similarly, IL-12 delivery from recombinant vaccinia virus attenuated the vector and enhanced the cellular immune response against HIV-1 Env in a dose-dependent manner. ${ }^{70}$

In another study, mucosal immunization of vacciniaimmune BALB/C mice with recombinant vaccinia expressing HIV gp160 induced specific serum antibody and strong HIV-specific cytotoxic $\mathrm{T}$ lymphocyte responses. These responses occurred not only in mucosal but also in systemic lymphoid tissue. The systemic HIVspecific cytotoxic $\mathrm{T}$ lymphocyte response was enhanced by coadministration of IL-12 at the mucosal site. ${ }^{71}$

When HIV-1 envelope DNA vaccine and interleukin 15 (IL-15) expression plasmid were intranasally administered to mice, a significant increase in the HIV-1-specific DTH response and CTL activity, and decrease in the serum $\operatorname{IgG} / \operatorname{IgG} 2$ a ratio was observed in the group which received DNA vaccine and IL-15 expression plasmid compared to DNA vaccination alone. Restimulated immune lymphoid cells from mice that received both agents showed enhanced production of interferongamma (IFN-gamma) and reduced secretion of IL-4. ${ }^{72}$

The importance of secretory $\operatorname{IgA}$ at mucosal surfaces was discussed earlier. A study describing an increase in specific local $\operatorname{Ig} \mathrm{A}$ responses to heterologous antigen in the lungs when genes for murine IL-5 and IL-6 in recombinant vaccinia virus were co-expressed and mice were inoculated intranasally, points out that an opportunity may exist to specifically increase the desired immunoglobulins in the mucosa. ${ }^{73}$ In another attempt to increase intestinal immune responses BALB/c mice were immunized with HIV-DNA vaccine formulated with a polymer, low-viscosity carboxymethylcellulose sodium salt (CMCS-L) via the intranasal (in.) route. The combination with the polymer elicited higher levels of antigen-specific serum IgG and fecal IgA antibodies than DNA vaccine alone and HIV-specific delayed-type hypersensitivity response and cytotoxic $\mathrm{T}$ lymphocyte activity were enhanced..$^{74}$ Mucosal secretory IgA can also be enhanced by co-inoculating MIP-1 $\alpha$ expression plasmid with the DNA vaccine. ${ }^{75}$

As genetic engineering evolves, it opens exciting possibilities to create a completely safe and effective vaccine, to boost specific immune responses at desired sites, and/or to enhance the desired effects, thus limiting the unwanted reaction of the mucosal tissue.

\section{References}

1 Brandtzaeg P, Baekkevold ES, Farstad IN et al. Regional specialization in the mucosal immune system: what happens in the microcompartments? Immunol Today 1999; 20: 141-151.

2 Persson CG, Erjefalt JS, Greiff L et al. Contribution of plasmaderived molecules to mucosal immune defence, disease and repair in the airways. Scand J Immunol 1998; 47: 302-13.

3 Brandtzaeg P. Mucosal immunity in the female genital tract. $J$ Reprod Immunol 1997; 36: 23-50.

4 Mascola J, Stiegler G, VanCott TC et al. Protection of macaques against vaginal transmission of a pathogenic HIV-1/SIV chimeric virus by passive infusion of neutralizing antibodies. Nat Med 2000; 6: 207-210.

5 Mascola J, Lewis MG, Stiegler G et al. Protection of macaques against pathogenic simian/human immunodeficiency virus 89.6PD by passive transfer of neutralizing antibodies. J Virol 1999; 73: 4009-4018.

6 Baba T, Liska V, Hofmann-Lehmann R et al. Human neutralizing monoclonal antibodies of the IgG1 subtype protect against mucosal simian-human immunodeficiency virus infection. Nat Med 2000; 6: 200-206

7 Robert-Guroff, M. IgG surfaces as an important component in mucosal protection. Nat Med 2000; 6: 129-130.

8 Kantele A, Zivny J, Hakkinen $\mathrm{M}$ et al. Differential homing commitments of antigen-specific $\mathrm{T}$ cells after oral or parenteral immunization in humans. J Immunol 1999; 162: 5173-5177.

9 Noll A, Bucheler N, Bohn E et al. DNA immunization confers systemic, but not mucosal, protection against enteroinvasive bacteria. Eur J Immunol 1999; 29: 986-996.

10 Okada E, Sasaki S, Ishii N et al. Intranasal immunization of a DNA vaccine with IL-12- and granulocyte- macrophage colonystimulating factor (GM-CSF)-expressing plasmids in liposomes induces strong mucosal and cell-mediated immune responses against HIV-1 antigens. J Immunol 1997; 159: 3638-3647.

11 Chen SC, Jones DH, Fynan EF. et al. Protective immunity induced by oral immunization with a rotavirus DNA vaccine encapsulated in microparticles. J Virol 1998; 72: 5757-5761.

12 Brandtzaeg P, Farstad IN, Haraldsen G. Regional specialization in the mucosal immune system: primed cells do not always home along the same track. Immunol Today 1999; 20: 267-277.

13 Mestecky J, Fultz PN. Mucosal immune system of the human genital tract. J Infect Dis 1999; 179 (Suppl 3): S470-S474.

14 Crowley-Nowick PA, Bell MC, Brockwell R. et al. Rectal immunization for induction of specific antibody in the genital tract of women. J Clin Immunol 1997; 17: 370-379.

15 Wang B, Dang K, Agadjanyan MG, et al. Mucosal immunization with a DNA vaccine induces immune responses against HIV-1 at a mucosal site. Vaccine 1997; 15: 821-825.

16 Livingston JB, Lu S, Robinson H, Anderson DJ et al. Immunization of the female genital tract with a DNA-based vaccine. Infect Immun 1998; 66: 322-329.

17 Westermann J, Bode U, Pabst R et al. Migration of so-called naive and memory $\mathrm{T}$ lymphocytes from blood to lymph in the rat. The influence of IFN-gamma on the circulation pattern. $J$ Immunol 1994; 152: 1744-1750.

18 Westermann J, Pabst R. How organ-specific is the migration of 'naive' and 'memory' T cells? [see comments]. Immunol Today 1996; 17: 278-282.

19 Drayson MT. The entry of lymphocytes into stimulated lymph nodes. The site of selection of alloantigen-specific cells. Transplantation 1986; 41: 745-751.

20 Fynan EF, Webster RG, Fuller DH, Haynes JR, Santoro JC, Robinson HL. DNA vaccines: protective immunizations by parenteral, mucosal, and gene- gun inoculations. Proc Natl Acad Sci USA 1993; 90: 11478-11482.

21 Condon C, Watkins SC, Celluzzi CM, Thompson K, Falo LD Jr. DNA-based immunization by in vivo transfection of dendritic cells. Nat Med 1996; 2: 1122-1128.

22 Ulmer JB, Deck RR, Dewitt CM, Donnhly JI, Liu MA. Generation of MHC class I-restricted cytotoxic T lymphocytes by expression 
of a viral protein in muscle cells: antigen presentation by nonmuscle cells. Immunology 1996; 89: 59-67.

23 Raz E, Tighe H, Sato Y et al. Preferential induction of a Th1 immune response and inhibition of specific IgE antibody formation by plasmid DNA immunization. Proc Natl Acad Sci USA 1996: 93: 5141-5145.

24 Martinez X, Brandt C, Saddallah F et al. DNA immunization circumvents deficient induction of $\mathrm{T}$ helper type 1 and cytotoxic T lymphocyte responses in neonates and during early life. Proc Natl Acad Sci USA 1997; 94: 8726-8731.

25 Haensler J, Verdelet C, Sanchez V et al. Intradermal DNA immunization by using jet-injectors in mice and monkeys. Vaccine 1999; 17: 628-638.

26 Raz E, Carson DA, Parker SE et al. Intradermal gene immunization: the possible role of DNA uptake in the induction of cellular immunity to viruses. Proc Natl Acad Sci USA 1994; 91: 9519-9523.

27 Ishida T, Mizushima Si, Azuma S, Kobayashi N et al. Identification of TRAF6, a novel tumor necrosis factor receptor- associated factor protein that mediates signaling from an amino- terminal domain of the CD40 cytoplasmic region. J Biol Chem 1996; 271: 28745-28748.

28 Charo J, Ciupitu AM, Le Chevalier De Preville A et al. A longterm memory obtained by genetic immunization results in full protection from a mammary adenocarcinoma expressing an EBV gene. I Immunol 1999; 163: 5913-5919.

29 Roy K, Mao HQ, Huang SK, Leong KW. Oral gene delivery with chitosan-DNA nanoparticles generates immunologic protection in a murine model of peanut allergy. Nat Med 1999; 5: 387-391.

30 Bona CA, Casares S, Brumeanu TD. Towards development of T-cell vaccines. Immunol Today 1998; 19: 126-133.

31 Roscoe DE, Holste WC, Sorhage FE et al. Efficacy of an oral vaccinia-rabies glycoprotein recombinant vaccine in controlling epidemic raccoon rabies in New Jersey. J Wildl Dis 1998; 34: 752763.

32 Kent SJ, Zhao A, Best SJ, Chandler JD, Boyle DB, Ramshaw IA. Enhanced T-cell immunogenicity and protective efficacy of a human immunodeficiency virus type 1 vaccine regimen consisting of consecutive priming with DNA and boosting with recombinant fowlpox virus. J Virol 1998; 72: 10180-10188.

33 Hanke T, Samuel RV, Blanchard TJ et al. Effective induction of simian immunodeficiency virus-specific cytotoxic $\mathrm{T}$ lymphocytes in macaques by using a multiepitope gene and DNA prime- modified vaccinia virus Ankara boost vaccination regimen. J Virol 1999; 73: 7524-7532.

34 Caver TE, Lockey TD, Srinivas RV, Webster RG, Hurwitz JL. A novel vaccine regimen utilizing DNA, vaccinia virus and protein immunizations for HIV-1 envelope presentation. Vaccine 1999; 17: 1567-1572.

35 Chatterjee S, Qin H, Manna S, Tripathi PK, Recombinant vaccinia virus expressing GM-CSF as tumor vaccine. Anticancer Res 1999; 19: 2869-2873.

36 Valmori D, Levy F, Miconnet I et al. Induction of potent antitumor CTL responses by recombinant vaccinia encoding a melan-A peptide analogue. J Immunol 2000; 164: 1125-1131.

37 Paoletti E. Poxvirus recombinant vaccines. Ann NY Acad Sci 1990; 590: 309-325.

38 Franchini G, Bosch ML. Genetic relatedness of the human immunodeficiency viruses type 1 and 2 (HIV-1, HIV-2) and the simian immunodeficiency virus (SIV). Ann NY Acad Sci 1989; 544: $81-87$.

39 Robert-Guroff M, Aldrich K, Muldoon R et al. Cross-neutralization of human immunodeficiency virus type 1 and 2 and simian immunodeficiency virus isolates. J Virol 1992; 66: 3602-3608.

40 Abimiku A, Franchini G, Tatrtaglia J et al. HIV-1 recombinant poxvirus vaccine induces cross-protection against HIV-2 challenge in rhesus macaques. Nat Med 1995; 1: 321-329.

41 Graham BS, McElrath MJ, Connor RI et al. Analysis of intercurrent human immunodeficiency virus type 1 infections in phase I and II trials of candidate AIDS vaccines. AIDS Vaccine Evalu- ation Group, and the Correlates of HIV Immune Protection Group. J Infect Dis 1998; 177: 310-319.

42 Chengalvala M, Lubeck MD, Davis AR et al. Evaluation of adenovirus type 4 and type 7 recombinant hepatitis $B$ vaccines in dogs. Vaccine 1991; 9: 485-490.

43 Schneider M, Graham FL, Prevec L. Expression of the glycoprotein of vesicular stomatitis virus by infectious adenovirus vectors. J Gen Virol 1989; 70: 417-427.

44 Lubeck MD, Natuk R, Myagkikh M et al. Long-term protection of chimpanzees against high-dose HIV-1 challenge induced by immunization. Nat Med 1997; 3: 651-658.

45 Donson A, Foreman NK. Adenovirus mediated gene therapy in a glioblastoma vaccine model; specific antitumor immunity and abrogation of immunosuppression. J Neurooncol 1998; 40: 205214.

46 Xiang Z, Ertl HC. Induction of mucosal immunity with a replication-defective adenoviral recombinant. Vaccine 1999; 17: 2003-2008.

47 Ochsenbein A, Karrer U, Klenerman P. A comparison of T cell memory against the same antigen induced by virus versus intracellular bacteria. Proc Natl Acad Sci USA 1999; 96: 9293-9298.

48 Letvin NL, Daniel MD, Sehgal PK et al. Induction of AIDS-like disease in macaque monkeys with T-cell tropic retrovirus STLVIII. Science 1985; 230: 71-73.

49 Crotty S, Lohman BL, Lu FX, Tang S, Miller CJ, Andino R. Mucosal immunization of cynomolgus macaques with two serotypes of live poliovirus vectors expressing simian immunodeficiency virus antigens: stimulation of humoral, mucosal, and cellular immunity. J Virol 1999; 73: 9485-9495.

50 Todryk S, McLean C, Ali S et al. Disabled infectious single-cycle herpes simplex virus as an oncolytic vector for immunotherapy of colorectal cancer. Hum Gene Ther 1999; 10: 2757-2768.

51 Leitner W, Ying H, Driver DA, Dubensky TW, Restflo NP. Enhancement of tumor-specific immune response with plasmid DNA replicon vectors. Cancer Res 2000; 60: 51-55.

52 Kremer L, Dupre L, Riveau G, Capron A, Locht C. Systemic and mucosal immune responses after intranasal administration of recombinant Mycobacterium bovis bacillus Calmette-Guerin expressing glutathione S-transferase from Schistosoma haematobium. Infect Immun 1998; 66: 5669-5676.

53 Lagranderie M, Winter N, Balazuc AM, Gicquel B, Gheorghiu M. A cocktail of Mycobacterium bovis BCG recombinants expressing the SIV Nef, Env, and Gag antigens induces antibody and cytotoxic responses in mice vaccinated by different mucosal routes. AIDS Res Hum Retroviruses 1998; 14: 1625-1633.

54 Lagranderie M, Balazuc AM, Gicquel B, Gheorghiu M. Oral immunization with recombinant Mycobacterium bovis BCG simian immunodeficiency virus nef induces local and systemic cytotoxic T- lymphocyte responses in mice. J Virol 1997; 71: 2303-2309.

55 Winter N, Lagranderie M, Rauzier J et al. Expression of heterologous genes in Mycobacterium bovis BCG: induction of a cellular response against HIV-1 Nef protein. Gene 1991; 109: 47-54.

56 Kameoka M, Nishino Y, Matsuo K et al. Cytotoxic T lymphocyte response in mice induced by a recombinant BCG vaccination which produces an extracellular alpha antigen that fused with the human immunodeficiency virus type 1 envelope immunodominant domain in the V3 loop. Vaccine 1994; 12: 153-158.

57 Sizemore DR, Branstrom AA, Sadoff JC. Attenuated Shigella as a DNA delivery vehicle for DNA-mediated immunization. Science 1995; 270: 299-302.

58 Fennelly GJ, Khan SA, Abadi MA, Wild TF, Bloom BR. Mucosal DNA vaccine immunization against measles with a highly attenuated Shigella flexneri vector. J Immunol 1999; 162: 1603-1610.

59 Kaufmann S. Fundamental Immunology. Paul WE (ed). Lippincott-Raven: 1998, pp 1335-1371.

60 Darji A, Guzman CA, Gerstel B et al. Oral somatic transgene vaccination using attenuated S. typhimurium. Cell 1997; 91: 765-775.

61 Paglia P, Medina E, Arioli I, Guzman CA, Colombo MP. Gene transfer in dendritic cells, induced by oral DNA vaccination 
with Salmonella typhimurium, results in protective immunity against a murine fibrosarcoma. Blood 1998; 92: 3172-3176.

62 Pasetti MF, Anderson RJ, Noriega FR, Levine MM, Sztein MB. Attenuated deltaguaBA Salmonella typhi vaccine strain CVD 915 as a live vector utilizing prokaryotic or eukaryotic expression systems to deliver foreign antigens and elicit immune responses. Clin Immunol 1999; 92: 76-89.

63 Catmull J, Wilson ME, Kirchhoff LV, Metwali A, Donelson JE. Induction of specific cell-mediated immunity in mice by oral immunization with Salmonella expressing Onchocerca volvulus glutathione S-transferase. Vaccine 1999; 17: 31-39.

64 Dietrich G, Bubert A, Gentschev I et al. Delivery of antigen-encoding plasmid DNA into the cytosol of macrophages by attenuated suicide Listeria monocytogenes [see comments]. Nat Biotechnol 1998; 16: 181-185.

65 Maassen C, Laman JD, den Bak-Glashouwer MJ et al. Instruments for oral disease-intervention strategies: recombinant Lactobacillus casei expressing tetanus toxin fragment $C$ for vaccination or myelin proteins for oral tolerance induction in multiple sclerosis. Vaccine 1999; 17: 2117-2128.

66 Sin JI, Kim JJ, Boyer JD, Ciccarelli RB, Higgins TJ, Weiner DB. In vivo modulation of vaccine-induced immune responses toward a Th1 phenotype increases potency and vaccine effectiveness in a herpes simplex virus type 2 mouse model. J Virol 1999; 73: 501-509.

67 Sin JI, Kim JJ, Arnold RL, Shroff KE et al. IL-12 gene as a DNA vaccine adjuvant in a herpes mouse model: IL-12 enhances Th1type CD4+ T cell-mediated protective immunity against herpes simplex virus-2 challenge. J Immunol 1999; 162: 2912-2921.

68 Tartaglia J, Franchini G, Robert-Guroff M et al. Highly attenuated poxvirus vector strains, NYVAC and ALVAC, in retrovirus vaccine development. In: Huitiëme Colloque Des Cent Gardes 1993 (Proceedings). Robert O (ed). Foundation Merieux: Paris, 1993, pp 293-298.

69 Benson J, Chougnet C, Robert-Guroff M, Montefiori D et al. Recombinant vaccine-induced protection against the highly pathogenic simian immunodeficiency virus SIV(mac251): dependence on route of challenge exposure. J Virol 1998; 72: 4170-4182.

70 Gherardi MM, Ramirez JC, Rodriguez D et al. IL-12 delivery from recombinant vaccinia virus attenuates the vector and enhances the cellular immune response against HIV-1 Env in a dose- dependent manner. J Immunol 1999; 162: 6724-6733.

71 Belyakov IM, Moss B, Strober W, Berzofsky JA et al. Mucosal vaccination overcomes the barrier to recombinant vaccinia immunization caused by preexisting poxvirus immunity. Proc Natl Acad Sci USA 1999; 96: 4512-4517.

72 Xin KQ, Hamajima K, Sasaki S et al. IL-15 expression plasmid enhances cell-mediated immunity induced by an HIV-1 DNA vaccine. Vaccine 1999; 17: 858-866.

73 Ramsay AJ, Leong KH, Boyle D, Ruby J, Ramshaw IA. Enhancement of mucosal IgA responses by interleukins 5 and 6 encoded in recombinant vaccine vectors. Reprod Fertil Dev 1994; 6: 389-392.

74 Hamajima K, Sasaki S, Fukushima J et al. Intranasal administration of HIV-DNA vaccine formulated with a polymer, carboxymethylcellulose, augments mucosal antibody production and cell- mediated immune response. Clin Immunol Immunopathol 1998; 88: 205-210.

75 Lu Y, Xin KQ, Hamajima K, Tsuji T. Macrophage inflammatory protein-1alpha (MIP-1alpha) expression plasmid enhances DNA vaccine-induced immune response against HIV-1. Clin Exp Immunol 1999; 115: 335-341. 